\title{
Ups and Downs of Nuclear Isomers
}

\author{
Isomers are set apart from other nuclear excitations by their \\ long half-lives. That longevity facilitates the study of nuclear \\ structure and astrophysics, and it suggests a variety of \\ practical applications.
}

exceptional combination of high excitation energy and conveniently long halflife has led to claims for practical applications that have lent the hafnium isomer unusual visibility (see PHYSICS TODAY, May 2004, page 21)

Nature's sole example of an isomer long-lived enough to be called stable is ${ }^{180 \mathrm{~m}} \mathrm{Ta}$. This tantalum isomer's

\section{Philip M. Walker and James J. Carroll}

W hat could one do with a clean source of nuclear energy? That tantalizing question reflects a dream that some have hoped to realize by exploiting the energy-storage capabilities of nuclear isomers. But the hope hinges on facets of nuclear behavior that remain unknown despite decades of study. Electromagnetic transitions and $\beta$ decay, the basic mechanisms that largely determine isomer halflives, are well understood. In many cases, however, it is still not possible to predict half-lives even to within an order of magnitude.

Today's isomer research seeks a better understanding of the degrees of freedom that will reveal new aspects of nuclear structure and lead the way to new applications. In this article, we lay out the essential ingredients of nuclear isomerism and take a look at future possibilities.

\section{Metastable excited states}

The term "isomer" is borrowed from chemistry, where it refers to molecules that have different geometrical configurations of the same collection of atoms. Isomeric nuclei, as distinguished from isotopes, are different states of the same numbers of protons and neutrons. Whereas chemical isomers have energy states that are similar, sometimes identical, to each other, nuclear isomers always have different energies. Excitation energies can be as high as several $\mathrm{MeV}$.

An interesting example of a nuclear isomer is ${ }^{99 \mathrm{~m}} \mathrm{Tc}$, an excited state of technetium-99. The " $\mathrm{m}$ " after the mass number denotes a metastable state-that is, a long-lived isomer. The half-life of ${ }^{99 \mathrm{~m}} \mathrm{Tc}$ is six hours and its excitation energy above the nuclear ground state of ${ }^{99} \mathrm{Tc}$ is $143 \mathrm{keV}$. By contrast, typical half-lives of excited nuclear states are about a picosecond. Isomers live at least a thousand times longer. The appellation is usually reserved for excited nuclear states that live longer than a nanosecond. The superscript " $\mathrm{m}$ " is even more restrictive; it's reserved for isomers with half-lives of more than a millisecond. If a nuclear species has more than one metastable isomer, an ordinal number after the " $\mathrm{m}$ " distinguishes between them in ascending order of excitation energy.

Perhaps the most widely known nuclear isomer is the long-lived hafnium excitation ${ }^{178 \mathrm{~m} 2} \mathrm{Hf}$. With a half-life of 31 years, it sits $2.4 \mathrm{MeV}$ above the stable ${ }^{178} \mathrm{Hf}$ ground state. The

Philip Walker is a professor of physics at the University of Surrey in England. James J. Carroll is a professor of physics at Youngstown State University in Ohio. half-life exceeds $10^{15}$ years. One can only quote a lower lifetime limit, because the isomer's spontaneous decay has never been observed. But the isomer sits $77 \mathrm{keV}$ above a ground state that is itself unstable, with a $\beta$-decay halflife of only eight hours.

Isomers can lose their excess energy by the usual radioactive decay modes: $\alpha, \beta$, or $\gamma$. The favored mode in any particular case depends on the energies and quantum numbers of the states involved. Decay by neutron or proton emission, or even by fission, is possible for some isomers.

What can isomers reveal about nuclei and about the environments - for example, sites of astrophysical nucleosynthesis-in which they are formed? How can nuclear isomers be put to practical uses here on Earth?

\section{Isomers in the clinic}

The nuclear isomer that is most often ingested for clinical purposes is ${ }^{99 \mathrm{~m}} \mathrm{Tc}$. It has excellent properties for medical imaging: a convenient half-life of six hours and an equally convenient soft-gamma-ray decay energy of $141 \mathrm{keV}$. Another useful feature is technetium's ability to bind chemically with many biomolecules. Furthermore, the isomer is easy to produce. One can, for example, bombard molybdenum-98 with neutrons from a reactor to make ${ }^{99} \mathrm{Mo}$, which then $\beta$-decays with a half-life of 66 hours to ${ }^{99 \mathrm{~m}} \mathrm{Tc}$. The technetium isomer is probably the most widely used of all radioactive isotopes for medical diagnostics. It is used to image the brain, bones, heart, lungs, and other organs (see figure 1).

It's not, in fact, the emission of the $141-\mathrm{keV}$ gamma that gives ${ }^{99 \mathrm{~m}} \mathrm{Tc}$ its desirably long half-life. The six hours are taken almost entirely by the first step in the isomer's descent to the ground state. That preliminary step is a 2 -keV decay by electron conversion, in which nuclear excitation energy is carried away by the ejection of an orbital electron from the atom. The subsequent emission of the 141-keV gamma from the nucleus takes less than a nanosecond.

The long half-life of the first step of ${ }^{99 \mathrm{~m}} \mathrm{Tc}$ decay is attributable to the isomer's angular momentum. Its spin $J$ and parity $\pi$ are $J^{\pi}=1 / 2^{-}$. But the state $2 \mathrm{keV}$ below the isomer has $J^{\pi}=7 / 2^{+}$. Therefore the initial $2-\mathrm{keV}$ electromagnetic step between those two states is governed by an electric-octupole (E3) transition-matrix element. A direct $143-\mathrm{keV}$ transition from the isomer straight to the $J^{\pi}=9 / 2^{+}$ ground state would have magnetic-hexadecapole (M4) character. Such high-multipole electromagnetic transitions are very slow. 
Figure 1. Medical imaging with the technetium isomer ${ }^{99 \mathrm{~m}} \mathrm{Tc}$ shows a front view of a patient with septic arthritis in the left knee. The widely used isomer decays with a half-life of six hours by emitting a 141-keV photon. The isomeric material, bound to a ligand compound, was injected intravenously. The ligand preferentially seeks out metabolically active sites such as the inflamed knee in this case. The radioactive emission was mapped by a gamma camera whose crystalline sodium iodide scintillator elements scan around the patient.

Another unusual aspect of ${ }^{99 \mathrm{~m}} \mathrm{Tc}$ is that technetium $(Z=43)$ has no stable nuclei. Its longest-lived isotope, ${ }^{98} \mathrm{Tc}$, has a half-life of 4 million years. Therefore it's not found in natural deposits on Earth. It was the first new element to be produced artificially (in 1937). Hence its name, from the Greek technetos, meaning artificial. Promethium $(Z=61)$, the only other element lighter than lead that has no stable isotopes, was first made in the laboratory in 1945.

\section{Isomers in the stars}

Because Tc has no stable isotopes, it is especially interesting for astrophysics. From observations of its lines in atomic spectra of red-giant stars, one finds that Tc is being made in such stars by nuclear reactions between other elements. Visible light from Tc atoms far away brings to us the most compelling evidence of nature's ongoing cosmic alchemy.

The study of naturally occurring radionuclides surviving from Earth's formation has provided the most accurate information about the age of our planet-about 4.6 billion years. Nuclides with half-lives of much less than a billion years are now at low or vanishing abundance. For example, uranium-238, with a half-life of $4.47 \times 10^{9}$ years, is now the most abundant uranium isotope by far. But ${ }^{235} \mathrm{U}$, on which most fission power depends, has a half-life of only $7.04 \times 10^{8}$ years and an abundance of $0.7 \%$ relative to ${ }^{238} \mathrm{U}$. If Earth had formed with equal abundances of each, the ${ }^{235} \mathrm{U} /{ }^{238} \mathrm{U}$ abundance ratio would now be $2.4 \%$. Therefore, there must have been several times more ${ }^{238} \mathrm{U}$ to start with. But that starting point depends on the details of stellar nucleosynthesis.

Nuclear isomers probe this astrophysical alchemy and the stellar environments in which it takes place. With its half-life much longer than the $10^{10}$-year age of the cos$\operatorname{mos},{ }^{180 \mathrm{~m}} \mathrm{Ta}$ is the only naturally occurring isomer that's stable on the scale of stellar lifetimes. But many isomers that $\beta$-decay on much shorter time scales can serve as useful stellar probes.

The exceptional half-life of ${ }^{180 \mathrm{~m}} \mathrm{Ta}$, like that of ${ }^{99 \mathrm{~m}} \mathrm{Tc}$, hinges on angular momentum. This time, though, the isomer's own high spin is the impediment. Its spin-parity state is $9^{-}$. A short-lived excited state $37 \mathrm{keV}$ below the iso- mer has $J^{\pi}=2^{+}$, and the nuclear ground state is $1^{+}$. Therefore, electromagnetic transitions from the isomer, if they exist, would have E7 and M8 character. But such high-multipole transitions have never been seen.

For tantalum in stars, the issue is the $\beta$-decaying ground state of ${ }^{180} \mathrm{Ta}$. If the star is hot enough (above $10^{9} \mathrm{~K}$ ), excitation and de-excitation of nuclei by thermal photons will yield an equilibrium mixture of the ground and isomeric states. That's the kind of environment required for slow-neutron-capture (s-process) nucleosynthesis (see the article by John Cowan and Friedrich-Karl Thielemann in PHYSICS TODAY, 0ctober 2004, page 47).

If one of the nuclear states in such an equilibrium $\beta$ decays with a short half-life, then the longer-lived state will be depleted-that is, its effective half-life is reduced. The case of ${ }^{180} \mathrm{Ta}$ is of particular interest because the transition probabilities for $\gamma$-excitation to higher-lying states from which it can decay have recently been measured. ${ }^{1}$ From those measurements, and the fact that ${ }^{180 \mathrm{~m}}$ Ta exists on Earth (albeit with the lowest abundance of any stable nuclide), one can infer that s-process production of the isomer takes place in stars at modest temperatures less than $3 \times 10^{8} \mathrm{~K}$.

However, the actual mechanism by which ${ }^{180 \mathrm{~m}} \mathrm{Ta}$ is synthesized remains a mystery. Proton capture and neutrino reactions are two possibilities. The name tantalum is particularly appropriate to the ongoing challenge of puzzling out the isomer's origins. The mythological Tantalus, miscreant son of Zeus, is perpetually punished by being kept just out of reach of food and water.

\section{A central role}

Isomers were first foreseen by Fredrick Soddy, long before the discovery of the neutron. In 1917 he wrote, "We can have isotopes with identity of atomic weight, as well as of chemical character, which are different in their stability and mode of breaking up." Otto Hahn is usually credited with the first experimental observation of isomers, in uranium salts in 1921. But the key observations came in 1935, when nuclear physicists in the Soviet Union and Britain found isomers in bromine and indium. ${ }^{2}$ These discoveries were soon followed by Carl von Weizsacker's theoretical explanation in terms of angular-momentum quantum numbers. ${ }^{3}$ He was describing "spin traps," which turn out to be the principal class of isomers. Spin traps relax very slowly because high-multipole electromagnetic transition amplitudes are intrinsically weak.

The explanation for the existence of high-spin states came from the formulation of the nuclear shell model in 
1949. Indeed, the existence of isomers demanded the shell model. Individual nucleon orbits must be able to carry high angular momentum, and the existence of isomers depends on the quantum numbers of those individual orbits. Notwithstanding this strong tie to the single-particle emphasis of the shell model, isomers also provided essential information for the collective aspects of nucleons in the nucleus. In 1953, Aage Bohr and Ben Mottelson ${ }^{4}$ interpreted the energies of the ${ }^{180} \mathrm{Hf}$ states one sees following the decay of ${ }^{180 \mathrm{~m}} \mathrm{Hf}$ as being characteristic of a quantum rotor, with energies proportional to $J(J+1)$. Thus did isomers take their place at the center of nuclear-structure investigations.

In the $1960 \mathrm{~s}$, nuclear isomers provided the first examples of superdeformed nuclei; they were twice as long as they were wide. The superdeformed americium isomer ${ }^{242 \mathrm{~m}} \mathrm{Am}$, discovered in 1962 , decays by fission. ${ }^{5}$ At the end of the decade, the cobalt isomer ${ }^{53 \mathrm{~m}} \mathrm{Co}$ yielded the first observation of decay by direct proton emission. ${ }^{6}$ Those discoveries have paved the way to extensive investigations of the associated decay phenomena. ${ }^{7}$ No one has yet found an isomer that decays by neutron emission.

\section{Neutron-rich nuclides}

Let us focus on some recent developments and unsolved problems. Access to heavy nuclides on the neutron-rich side of the valley of stability is experimentally difficult, but highly desirable. Figure 2 shows this wide-open region of

\section{Box 1. Broken Pairs and Isomer Half-Lives}

$A^{\prime}$

l even-even nuclides (that is, those having even proton and neutron numbers) have ground states of zero total-angular-momentum quantum number $J$ and positive parity $\pi$. That's because all the single-particle nucleon orbits are paired, giving the state of lowest total energy. Here we neglect the contribution of collective rotation of the entire nucleus, which can in general add energy and angular momentum to that arising from the individual nucleon orbits (see box 2). Excitation of the nucleus may break up a pair of nucleons, with one or both being promoted to higher orbits. This excitation is called a two-quasiparticle state to emphasize the importance of the pairing interaction, which modifies the energy and wavefunction of the nuclear level.

The remaining even-even core of nucleons contributes no excitation energy or angular momentum. That role belongs to the quasiparticles. Suppose two quasiparticles (that is, two broken-pair nucleons) in different orbits each had $f^{\pi}=11 / 2^{+}$. The total angular momentum of the nucleus would come from their vector sum. In that case, $J^{\pi}$ could range from $0^{+}$to $11^{+}$, depending on the relative orientation of the quasiparticle spins. Thus, high nuclear spin can result from as few as two unpaired nucleons.

"Spin-trap" isomers occur in even-even nuclei in just this way, when excited states require large-multipole electromagnetic transitions for their decay. In ${ }^{178} \mathrm{Hf}$, for example, the 31 year $16^{+}$isomer has four-quasiparticle character-that is, one broken neutron pair and one broken proton pair. But spin-trap unknown nuclides. The physics objectives include the understanding of neutron skins of such nuclei-the outer volume into which the neutron distribution extends significantly beyond the proton distribution. One also wants to determine the structure of nuclides along the path of rapidneutron-capture (r-process) nucleosynthesis. Understanding the $r$ process could yield deep insight into supernova explosions. Because nuclear binding becomes very weak

isomers can also occur in odd-even or odd-odd nuclei. The nuclide ${ }^{180} \mathrm{Ta}$ is odd-odd. Its two-quasiparticle $1^{+}$ground state already has one unpaired proton and one unpaired neutron. The ultralong-lived $9^{-}$isomer ${ }^{180 \mathrm{~m} T a}$ also has two-quasiparticle character, but with very different relative orientations of the neutron and proton orbits. High angular momentum explains these isomers qualitatively. But quantitatively, the situation is less satisfactory.

Both the spherical nuclear shell model of orbiting nucleons and the deformed-shell (Nilsson) model, which allows for nonspherical shapes, predict the existence of isomers. But beyond these shell models of independently orbiting nucleons, there are additional interactions between the unpaired nucleons and the core, and between quasiparticles, that are hard to quantify. They lead to uncertainties on the order of $100 \mathrm{keV}$ in the predicted excitation energies of isomers. There is also significant uncertainty in the calculation of isomer decay rates to collective rotational or vibrational states of the core.

Although the bulk behavior of the wavefunctions of isomers and other excited states are relatively well understood, the full details are still missing. Because electromagnetic decay rates depend sensitively on the overlap between wavefunctions for initial and final states, small discrepancies on the wings of the wavefunctions can lead to very different results. Additional data will be required for sorting out the relative importance of the different mechanisms that contribute to isomer decay. 
with increasing neutron excess, mean-field theoretical models may turn out to be completely inadequate in the neutron-rich domain.

A promising way of producing and studying neutronrich nuclides and their excited states was pioneered at the GANIL laboratory in Caen, France, and the GSI laboratory in Darmstadt, Germany. At these heavy-ion accelerator facilities, projectile nuclei fragment in high-energy collisions with target nuclei, and one finds isomers amid the debris.

In a recent experiment at GSI, Monica Caamaño and coworkers bombarded a beryllium-9 target with stripped lead-208 nuclei accelerated to an energy of $1 \mathrm{GeV}$ per nucleon. ${ }^{8}$ The fragments of the $\mathrm{Pb}$ nucleus, after being tracked through a magnetic separator, were stopped and their $\gamma$-decay photons were measured in germanium detectors (see figure 3 ). The experimenters were able to associate the emitted gammas, ion by ion, with specific fragments over decay-sequence correlation times as long as $100 \mu \mathrm{s}$. In this way, Caamaño and company identified eleven new isomeric states of neutron-rich nuclides with mass numbers from 188 to 203 . The isomeric half-lives ranged from $10 \mathrm{~ns}$ to $1 \mathrm{~ms}$.

These neutron-rich nuclides are still some way from the neutron-skin regime and the r-process pathway. Nevertheless, such studies are paving the way to those longerterm objectives. At the same time, the experiments have already uncovered unexpected nuclear-structure effects. An important feature of isomers with half-lives in the microsecond region is the experimenter's ability to correlate fragments of the projectile nucleus with their decay products. A few microseconds is long enough to track and identify the fragments, yet short enough to distinguish timecorrelated decay events from accidental background correlations.

For longer-lived fragments, one can observe isomer and ground-state properties by injecting the fragments into a storage ring. ${ }^{9}$ In recent measurements of heavy, neutron-rich nuclei by the storage-ring technique, experimenters were seeking, among other things, a predicted $J^{\pi}=18^{+}$isomer of ${ }^{188} \mathrm{Hf}$ that might have intriguing similarities to the much-touted isomer ${ }^{178 \mathrm{~m} 2} \mathrm{Hf}$, whose spin-parity is $16^{+}$. The data are still under analysis.

The development of new, high-intensity projectilefragmentation facilities is now a key part of planning for the future of nuclear physics worldwide, with projects such as RIA (Rare Isotope Accelerator) in the US and FAIR (Facility for Antiproton and Ion Research) in Germany. While addressing a wide range of nuclear and applied-science issues, these new facilities will provide important new opportunities for the study of isomers, especially in heavy, neutron-rich nuclides.

\section{Superheavy nuclides}

The search for the heaviest possible elements is a quest of long standing. For the so-called superheavy elements- those with more than 100 protons-the strong nuclear force between adjacent nucleons is insufficient to overcome the longer-range Coulomb repulsion between the protons. Their existence relies on subtle shell-model effects on the orbits of protons and neutrons inside the nucleus. Such small effects can, in fact, yield long half-lives-but just how long remains uncertain. We don't know, for example, the true nature of the predicted "island of stability" at nuclear masses beyond the present experimental limits.

Superheavy nuclei can have isomer states. Indeed, such isomers can live longer than their ground states. ${ }^{10}$ Fission is often described by the liquid-drop model. A key feature of the superheavy isomers is that the nucleon-pair breaking which makes their formation possible also breaks down the superfluidity of the nucleon fluid inside the nucleus. It becomes viscous, and that slows down the fission process. The probability for $\alpha$-particle decay is also reduced by the pair breaking. For example, an isomer of darmstadtium-270 (the name was officially given to element 110 two years ago) has been found to have a half-life some 60 times longer than its ground state. Thus the predicted abundance of isomers in superheavy nuclei could be of great help in the exploration of that extreme frontier of the nuclear landscape.

\section{Vibration and rotation}

The total angular momentum of a nucleus has contributions from individual nucleon orbits and from collective rotation of the nucleus as a whole. Bonded pairs of orbits and, especially, closed shells of such pairs contribute no net angular momentum. In strongly deformed nuclei, nucleon numbers are far from shell closure, and collective rotation usually dominates the low-energy, high-angular-momentum structure. This happens when the orbits of unpaired nucleons cannot generate much angular momentum on their own. In that case, high total angular momentum results most easily (at low energies) from high collective rotation plus a lesser contribution from any unpaired orbits.

There is, however, a small region of nuclides around ${ }^{178} \mathrm{Hf}(Z=72$ and $N=106$, see figure 4$)$ where isomers are 
"yrast." The term, from the Swedish word for dizziest, refers to the lowest-energy nuclear state for a given angular momentum. For an yrast isomer, the contribution of collective rotation to the angular momentum is minimal. The isomers near ${ }^{178} \mathrm{Hf}$ are yrast as a result of high-spin nucleon orbitals close to the Fermi surface. That effect is well described by the deformed-shell model ${ }^{11}$ (see box 1).

Vibrational excitations of deformed nuclei are known to be energetically disfavored. They are far from the yrast states. Recent findings, however, have given evidence for a new type of energetically favored vibrational behavior based on isomers. This effect arises because the maximumangular-momentum vibrational states have energies proportional to $J$, whereas rotational energies depend quadratically on $J$. Thus, as angular momentum increases, rotational excitations become increasingly expensive relative to vibrational excitations. Lee Pattison (University of Manchester, UK) and coworkers have reported a dramatic consequence of this competition. ${ }^{12}$ On top of the $J^{\pi}=25^{+}$ isomer of osmium-182, they found a sequence of vibrational excitations that are, in fact, yrast. Their theoretical understanding of this structure is, thus far, based on a model of "tidal waves" that offers a new perspective on nuclear excitation modes.

\section{Isomer decay in deformed nuclei}

So far the discussion has concentrated on the utility of isomers for illuminating other nuclear phenomena. Now we take a closer look at isomer decay rates, focusing on socalled $K$ isomers in deformed nuclei. The quantum number $K$ is (in units of $\hbar$ ) the projection of the nuclear angular momentum on the symmetry axis of the nucleus (see box 2). The decay of isomers by electromagnetic transitions of multipole order lower than $\Delta K$, the difference in $K$ between initial and final states, requires a mixing of $K$ values in either state. This $K$ mixing need only have a small influence on the wave function to produce a dominant effect on the rates of otherwise forbidden de-excitation transitions.

Three distinct mechanisms have been proposed for the requisite $K$ mixing. First, there is Coriolis-force mixing in the noninertial rest frame of the rotating nucleus. One can view the Coriolis effect as causing fluctuations in the orientation of the nucleus without any fluctuation in its shape. Second, because $K$ conservation depends on axial symmetry, asymmetric wavefunction components can instigate $K$ mixing. That effect is equivalent to nuclear shape fluctuations with orientation fixed. Finally, there is statistical mixing: Quantum states of the same spin and parity, but different $K$, can mix significantly by virtue of fortuitous near-degeneracies between their energies. Such accidental degeneracies become more common with increasing density of energy levels. And the level density, in turn, grows with excitation energy.

Each of the three $K$-mixing mechanisms, on its own, has had some success in fitting the isomer data. ${ }^{7}$ But a unified approach has yet to be developed, and the relative importance of the different mechanisms remains to be determined. The situation leaves the prediction of some isomer half-lives uncertain by many orders of magnitude.

\section{Inducing premature decay}

Let's return to our opening question about clean energy sources. A special feature of nuclear isomers is that they carry excess energy. And that energy can be released through the electromagnetic interaction. Indeed, the energy release can be induced by photon bombardment. In other words, one can shorten the isomer's decay time and thus release the excitation energy in a controlled manner.

That much is well established. For example, the $10^{15}$. year isomer ${ }^{180 \mathrm{~m}} \mathrm{Ta}$ can be converted to the 8 -hour $\beta$-decaying ${ }^{180} \mathrm{Ta}$ ground state by photon bombardment, a process that has been studied in detail. ${ }^{1,13}$ But unfortu-

\section{Box 2. $K$ Isomers}

T he orientation of a deformed isomer's spin relative to the symmetry axis can strongly affect its half-life. Nuclei with magic (closed-shell) neutron or proton numbers of 2, 8, 20, $28,50,82$, or 126 (see figure 2) are more or less spherical in their ground states and low-energy excitations. But far away from closed shells, the shape is typically prolate, with welldefined symmetry about the long axis (see the illustration at right). A prolate nucleus can rotate collectively, but quantum mechanics forbids collective rotation about an axis of cylindrical symmetry. The total angular momentum of the nucleus is a vector sum of contributions from collective rotation and orbits of unpaired nucleons. Whatever their relative contributions, the total angular momentum's component $K \hbar$ along the symmetry axis is a constant of the motion.

In the noninertial rotating reference frame, however, $K$ is

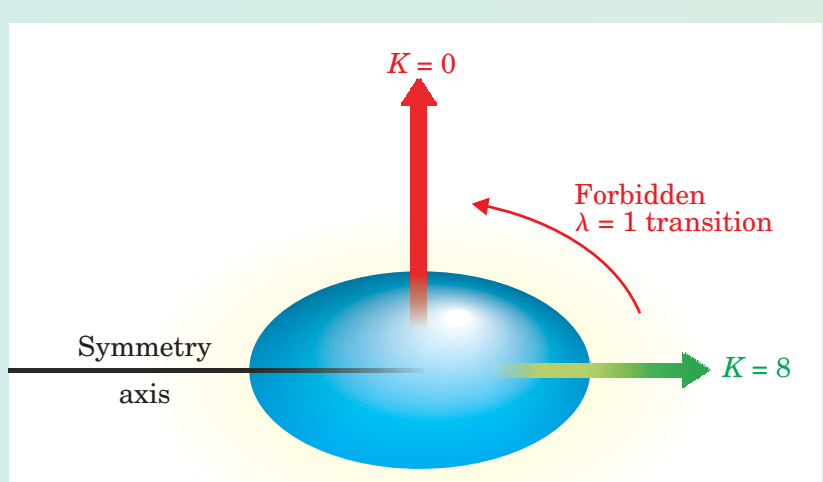

not strictly conserved, primarily because of the Coriolis effect. Just as cyclones-another Coriolis manifestation-have their angular momenta aligned with Earth's axis, nucleon orbits tend to become aligned with the nuclear rotation. The result is a mixing of different $K$ states.

The $K$ quantum number introduces a nuclear-transition selection rule based on the direction as well as the magnitude of the angular momentum: In an electromagnetic de-excitation transition, the change in $K$ should not exceed $\lambda$, the transition multipole order. Transitions that violate this condition are called $K$-forbidden. The drawing above illustrates a $K$-forbidden dipole $(\lambda=1)$ transition between prolate $J=8$ nuclear states. In the initial $K=8$ orientation, the angular momentum can be aligned with the symmetry axis because it arises completely from unpaired nucleon orbits rather than collective rotation. But in the lower-energy $K=0$ orientation, the $J=8$ angular momentum is entirely from collective rotation.

Rather than being strictly forbidden, such transitions are severely hindered. The result is long-lived excitation states-that is, nuclear isomers. These "K isomers" form a distinct class of isomers in axially symmetric deformed nuclei. Their distribution in the $N-Z$ plane of neutron and proton numbers (see figure 4), clustered around the 31 -year isomer of hafnium-178, is quite distinct from the two clusters of closed-shell (spin-trap) isomers near $N=82$ and $Z=82$. 
nately, the energy of the incident photon that liberates the isomer's 77-keV excitation energy has to exceed an MeV! Although this $\gamma$ induced de-excitation has interesting astrophysical implications, it hardly seems like the basis for a practical energy-storage device.

The scientific interest in such matters entered a new and controversial phase following claims made in 1999 that ${ }^{178 \mathrm{~m} 2} \mathrm{Hf}$ could release its $2.4 \mathrm{MeV}$ of stored energy when bombarded by x-ray photons with energies of less than $100 \mathrm{keV}$. If such triggering were indeed possible at a reasonable rate, the practical implications could have been major.

The ensuing complex story of claims and counterclaims has been told in an article by one of $\mathrm{us}^{14}$ and in the May 2004 PHYsICS TODAY news story cited above. We don't wish to repeat those arguments here. Suffice it to say that the original claims have not been confirmed to the satisfaction of the nuclear physics community. There are ongoing efforts to quantify the photon de-excitation of ${ }^{178 m} 2 \mathrm{Hf}$. The physics of isomer interaction with the radiation field implies that the de-excitation will almost certainly be possible at some photon energy. But that threshold energy, while of scientific interest, will very likely be too high to be of practical use. Of course, other isomers may prove themselves useful.

One can consider, for example, the possibility of stimulated emission from isomers, and even the construction of a $\gamma$-ray laser based on isomer decay. Line broadening, the most critical impediment to isomer lasing, might perhaps be overcome by exploiting the Mössbauer effect. But many other difficulties remain. ${ }^{15}$ Nevertheless, the potential applications of isomers are widespread, and a better understanding of the processes that control their decay may lead to key advances.

The atomic environment can have a large effect on nuclear decay rates-for example, through the electronconversion process. To take an extreme case: If all the electrons were stripped from a ${ }^{178} \mathrm{Hf}$ atom while its nucleus was in the $J^{\pi}=16^{+}$isomeric state, its half-life would increase from the usual 31 years to $10^{5}$ years. More promising for applications, of course, are techniques for hastening isomer decay. For example, a $20 \%$ half-life reduction has been demonstrated $^{16}$ for highly stripped ions of an isomer of iron-57. Less dramatic is the recently discovered $1 \%$ shortening of the ground-state half-life of ${ }^{7} \mathrm{Be}$ when the atoms are caged within $\mathrm{C}_{60}$ buckeyballs. ${ }^{17}$ The sensitivity of nuclear decay to the atomic environment gives hope that mixed atomic-nuclear effects may eventually provide a way to induce energy release from isomers.

Exploiting the practical promise of isomers will require a much improved understanding of their basic properties. Theorists Wojciech Satula (University of Warsaw) and Ramon Wyss (Royal Institute of Technology, Stockholm) have reviewed the considerable recent progress in the understanding of high-spin nuclear excitations. ${ }^{18}$ For

the experimental progress of nuclear physics, isomers are excellent tools for accessing species far from the valley of stability, particularly for the study of neutron-rich heavy nuclides, and even superheavy nuclides.

\section{References}

1. D. Belic et al., Phys. Rev. C 65, 035801 (2002).

2. B. Kurtchatov, I. Kurtchatov, L. Moussovski, L. Roussinov, Comptes Rendus Acad. Sci. 200, 1201 (1935); L. Szilard, T. A. Chalmers, Nature 135, 98 (1935).

3. C. F. von Weizsacker, Naturwissenschaften 24, 813 (1936).

4. A. Bohr, B. R. Mottelson, Phys. Rev. 90, 717 (1953).

5. S. M. Polikanov et al., Sov. Phys. JETP 15, 1016 (1962).

6. K. P. Jackson et al. Phys. Lett. B 33, 281 (1970).

7. P. M. Walker, G. D. Dracoulis, Nature 399, 35 (1999); Hyperfine Int. 135, 83 (2001).

8. M. Caamaño et al., Eur. Phys. J. A 23, 201 (2005).

9. Y. A. Litvinov et al., Phys. Lett. B 573, 80 (2003).

10. F. R. Xu, E. G. Zhao, R. Wyss, P. M. Walker, Phys. Rev. Lett. 92, 252501 (2004); S. Hofmann et al., Eur. Phys. J. A 10, 5 (2001).

11. K. Jain et al., Nucl. Phys. A 591, 61 (1995).

12. L. K. Pattison et al., Phys. Rev. Lett. 91, 182501 (2003).

13. P. M. Walker, G. D. Dracoulis, J. J. Carroll, Phys. Rev. C 64, 061302(R) (2001).

14. J. J. Carroll, Laser Phys. Lett. 1, 275 (2004).

15. G. C. Baldwin, J. C. Solem, Rev. Mod. Phys. 69, 1085 (1997); J. J. Carroll, S. Karamian, L. Rivlin, A. Zadernovsky, Hyperfine Int. 135, 3 (2001).

16. W. R. Phillips et al., Phys. Rev. Lett. 62, 1025 (1989).

17. T. Ohtsuki et al., Phys. Rev. Lett. 93112501 (2004).

18. W. Satula, R. A. Wyss, Rep. Prog. Phys. 68, 131 (2005). 